01

\title{
Аттестация поверочных газовых смесей с использованием современной спектроскопии: подход и применение
}

\author{
(ㄱ Л.А. Конопелько ${ }^{1,2}$, В.В. Белобородов ${ }^{1,2}$, Д.В. Румянцев ${ }^{1}$, Д.Н. Селюков ${ }^{3}$ \\ ${ }^{1}$ Всероссийский научно-исследовательский институт метрологии имени Д.И. Менделеева, \\ 190005 Санкт-Петербург, Россия \\ 2 Университет ИТМО, \\ 197101 Санкт-Петербург, Россия \\ 3 „МОНИТОРИНГ“, \\ 196247 Санкт-Петербург,Россия \\ e-mail: fhi@b10.vniim.ru
}

Поступила в редакцию 15.12 .2017 г.

Исследована возможность использования доступных спектроскопических баз данных для высокоточных измерений спектральных параметров газов. Предложен новый подход к измерению концентраций газовых компонентов $\left(\mathrm{HCl}, \mathrm{CO}, \mathrm{NO}, \mathrm{SO}_{2}\right)$ в газовых смесях с использованием спектроскопических баз данных HITRAN или GEISA. Разработан метод аттестации поверочных газовых смесей методом ИК фурьеспектроскопии для переноса единиц молярной доли на рабочие эталоны с целью уменьшения необходимого количества эталонов сравнения и повышения точности измерений.

DOI: $10.21883 /$ OS.2018.05.45937.289-17

\section{Введение}

Доступные спектроскопические базы данных и каталоги спектральных параметров газов собирались без участия метрологического сообщества. Это является причиной, почему современные спектральные оптические методы не реализованы в газовом анализе в полном объеме. Тем не менее, существует возможность использования доступных спектроскопических баз данных для высокоточных измерений. В настоящей работе представлен наш подход к измерению концентраций газовых компонентов $\left(\mathrm{HCl}, \mathrm{CO}, \mathrm{NO}, \mathrm{SO}_{2}\right)$ в газовых смесях с использованием спектроскопических баз данных HITRAN или GEISA. Метрологическая прослеживаемость обычно реализуется путем сравнения газовых смесей высокой точности (эталонов сравнения, ЭС), изготовленных в национальном первичном эталоне молярной доли и массовой концентрации компонентов в газовых средах ГЭТ 154-2016, и рабочих эталонов (в том числе газовых смесей). Количество требуемых ежегодно ЭС составляет около 1000 газовых смесей в баллонах, что является серьезным бременем для ГЭТ 154-2016.

Новый метод аттестации поверочных газовых смесей (ПГС) при помощи ИК фурье-спектроскопии, представленный в настоящей работе, не требует газовых смесей самой высокой точности - ЭС, так как он основан на спектроскопических параметрах газов. Однако использование имеющихся спектроскопических баз данных (БД) для метрологических приложений затруднено ввиду отсутствия бюджета неопределенности для спектроскопических параметров газов и несоблюдения других метрологических требований [1-3].
Современные спектральные приборы с использованием специальных компьютерных программ потенциально могут измерять концентрацию с высокой степенью точности, в частности на основе точных параметров газов спектроскопических БД. Тем не менее, отставание развития спектроскопических БД и спектральных методов от измерительных возможностей современных приборов приводит к потере точности и к усложненным методам анализа.

Измерение концентрации веществ, как правило, основано на сравнении измеренного спектра с эталонным спектром. В этой работе исследован метод получения эталонного спектра, который основан на вычислении спектра с использованием известных спектроскопических параметров молекул и спектральных характеристик аппаратуры. Среди доступных БД, используемых для численного моделирования спектров [4-10], в настоящее время наиболее широко используемыми являются HITRAN [7] и GEISA [8]. Существуют также каталоги сечений поглощения большого количества веществ, например, спектральный атлас Химического института Макса Планка в Майнце (MPI-Mainz-UV-VIS) [9] и количественная инфракрасная БД Национального института стандартов и технологий США (NIST) [10].

Вышеуказанные спектроскопические БД и каталоги, за исключением [10], не являются метрологически обеспеченными. БД NIST в настоящее время состоит только из набора 21-го спектра поглощения летучих органических соединений. Эти спектры были измерены с использованием газовых смесей, приготовленных и аттестованных в NIST.

Отсутствие метрологического обеспечения спектроскопических БД является причиной того, почему со- 
временные спектральные оптические методы не были в полной мере реализованы в газовом анализе.

Для измерения концентрации газа с использованием расчетного спектра необходимо соблюдение следующих требований.

1. Точное знание параметров спектральных линий.

2. Точное знание аппаратной функции и других технических данных ИК фурье-спектрометра.

Расчетные спектры могут отличаться от измеренных спектров, принятых для той же концентрации газа, изза неточности параметров спектральных линий, представленных в спектроскопических БД. Например, вычисленное изменение сигнала корреляционного газоанализатора типа „газовый фильтр“ (ГФ) [11] составляет около $1.3 \%$ [2] при изменении на 5\% интенсивности линии поглощения моноксида углерода $2171 \mathrm{~cm}^{-1}$ со спектральным разрешением $10 \mathrm{~cm}^{-1}$.

Имеются работы, посвященные сравнению БД HITRAN и GEISA [8,12]. Например, авторы [8] представляют сравнение значений интенсивности линий $\mathrm{H}_{2} \mathrm{O}$ в спектральном диапазоне (1400-2100) $\mathrm{cm}^{-1}$, приведенных в БД HITRAN и GEISA. Как следует из этого сравнения, разница интенсивности отдельных спектральных полос может достигать $\pm 10 \%$. Типичные неопределенности моделирования ИК спектров при использовании различных спектроскопических БД HITRAN-2008, GEISA-2011 и HITRAN-2012 оцениваются в [13]. Показано, что новейшие БД GEISA-2011 и HITRAN-2012 не устраняют несоответствия между экспериментальными и численными спектрами, найденными ранее, когда была использована в расчетах БД HITRAN-2008.

Обе БД - HITRAN и GEISA - имеют параметры, характеризующие точность (GEISA имеет больше таких параметров). По сути, эти БД являются сборниками спектроскопических параметров газов, полученных от различных групп исследователей. Эти группы не используют одни и те же сертифицированные и одобренные методы измерений, те же самые газовые смеси и аналогичное оборудование, как это принято в метрологическом сообществе. Группы исследователей приписывают измерениям некоторую точность независимо друг от друга.

Таким образом, для повышения точности спектроскопических БД необходимо введение методологии рутинных сличений, которая практикуется в национальных метрологических институтах разных стран и проводится под эгидой международного бюро мер и весов (МБМВ) [14-16]. Следует понимать, что создание признанной спектроскопической БД требует огромных усилий и времени с участием многих международных команд. Тем не менее, имеющиеся спектроскопические БД могут быть использованы для получения метрологически обеспеченных результатов измерений, как показано в настоящей работе. В этой работе мы проводим расчет поправочных коэффициентов $K$ для ограниченного числа газовых компонентов с использованием БД HITRAN только потому, что мы начали использовать ее раньше, и не потому, что БД HITRAN более точна, чем БД GEISA.

B настоящей работе мы представляем метод корректировки содержания целевых компонентов $(\mathrm{HCl}, \mathrm{CO}$, $\left.\mathrm{NO}, \mathrm{SO}_{2}\right)$ в газовых смесях, полученных с помощью спектроскопической БД HITRAN или GEISA. Таким образом, мы достигаем практических задач в передаче единиц от ГЭТ 154-2016 [17,18] к рабочим эталонам.

Для реализации этого метода мы разработали программное обеспечение, которое обеспечивает вычисление спектра монохроматического коэффициента пропускания с помощью параметров линий в БД HITRAN, свертку вычисленного монохроматического коэффициента пропускания спектра с аппаратной функцией прибора, и рассчитали содержание анализируемого компонента в газовой смеси с использованием вычисленного и измеренного спектров пропускания анализируемого компонента по методу наименьших квадратов.

Всероссийский научно-исследовательский институт метрологии имени Д.И.Менделеева (ВНИИМ) принимал участие в международных ключевых сличениях CCQM-P110-B2 [19], проведенных под эгидой МБМВ, в частности, для решения вопросов, связанных с измерениями концентрации газа с использованием вычисленного спектра. В дальнейшем были разработаны методы и процедуры измерения для передачи единиц измерений от ГЭТ 154-2016 к рабочим эталонам. Научные основы и методы практической реализации обсуждаются в настоящей работе.

\section{Методология. Научные основы передачи молярной доли от национального первичного эталона молярной доли и массовой концентрации компонентов в газовых средах (GET 154-2011) к рабочим эталонам с помощью ИК фурье-спектроскопии}

Метрологическая прослеживаемость обычно реализуется путем сравнения газовых смесей высокой точности (эталонов сравнения, ЭС), изготовленных в ГЭТ 154-2016, и рабочих эталонов. Количество требуемых ежегодно ЭС составляет около 1000 газовых смесей в баллонах, что является серьезным бременем для ГЭТ 154-2016.

Первая цель этой работы заключалась в разработке метода измерения для минимизации количества ЭС. Вторая цель этой работы заключалась в повышении точности переноса молярной доли от ГЭТ 154-2016 к рабочим эталонам. Эти цели достигнуты путем разработки методики измерений. ИК фурье-спектрометры (FTIR) были использованы в качестве измерительных приборов.

Имеется несколько возможных алгоритмов аттестации газовых смесей. 
Во-первых, это измерение концентрации анализируемого вещества в анализируемой газовой смеси путем градуировки с использованием эталонной газовой смеси при условии близости концентраций эталона и анализируемой газовой смеси, а также температуры и давления. Преимуществом этого метода является его независимость от условий измерения и аппаратной функции прибора. Недостатком этого метода является необходимость использования очень большого количества эталонных смесей.

Во-вторых, это измерение концентрации анализируемого вещества путем сравнения экспериментального спектра анализируемой газовой смеси с эталонным спектром в базе данных, соответствующим эталонной газовой смеси. Эталонный спектр представляет собой спектр известной концентрации одного газа. Например, большой список эталонных спектров представлен на сайте Gasmet [20]. В ходе реализации этого метода используются эталонные газовые смеси, спектр пропускания которых регистрируется один раз при определенных условиях. Только БД эталонных спектров газовых смесей или коэффициентов поглощения $\left(k_{i}\right)$ используется в этом случае. Преимуществом метода является меньшее потребление стандартных газовых смесей и более быстрый анализ.

К недостаткам этого метода следует отнести:

- ограниченное использование для расчета молярной доли анализируемого вещества в газовой смеси в узком диапазоне значений, в котором был определен коэффициент поглощения $\left(k_{i}\right)$ или был получен спектр;

- отсутствие учета влияния на коэффициент поглощения условий измерения: температуры и атмосферного давления;

- невозможность учесть изменение аппаратной функций прибора в процессе эксплуатации.

Для того чтобы разработать оптимальный алгоритм для определения содержания целевых компонентов, были проведены исследования методом расчета на основе спектроскопических БД.

Для определения параметров аппаратной функции прибора ИК фурье-спектрометра и контроля изменения аппаратной функции прибора должна периодически использоваться высокостабильная смесь тестового газа. Линии поглощения такого газа не должны перекрываться. СО в азоте в запечатанной оптической кювете хорошо подходит для этих целей. Для дальнейшего правильного расчета синтетического спектра поглощения с использованием параметров линий БД HITRAN важно знать условия измерения, т.е. давление $P$, температуpy $T$, а также длину тестовой оптической кюветы $L$. Производится вычисление синтетического спектра поглощения оптической кюветы с $\mathrm{CO}$ и подгонка наиболее приближенной модели аппаратной функции ИК фурьеспектрометра с использованием БД HITRAN.

В ходе реализации данного метода однократно используются первичные эталонные газовые смеси (ПЭГС) для получения поправочных коэффициентов. Для данных газовых смесей получают однократно спектры пропускания для известных условий $(P, T, L)$ и используют их при расчете поправочных коэффициентов с использованием БД HITRAN. Далее создается БД поправочных коэффициентов для диапазона номинальных концентраций используемых ПЭГС.

То есть алгоритм можно представить следующим образом:

(1) Экспериментальный спектр ПЭГС измеряется на конкретном ИК фурье-спектрометре с известной аппаратной функцией при известных условиях $(P, T, L)$ и известной концентрации ПЭГС $\left(C_{\text {pegm }}\right)$, записанной в паспорте ПЭГС.

(2) В то же время вычисление спектра выполняется с использованием параметров линии спектроскопической БД HITRAN для тех же самых условий измерения и той же концентрации ПЭГС $\left(C_{\text {pegm }}\right)$, которая используется для получения экспериментального спектра. Известные выражения расчета спектра пропускания с использованием лоренцева контура линии (выражения (11)-(17)), используемые в алгоритме, представлены в Приложении.

Концентрация газа в расчетном спектре $C_{\text {calc }}$ (как будто не зная, какая концентрация $C_{\text {pegm }}$ была введена в вычисления в качестве исходной) определяется с использованием экспериментального спектра ПЭГС в качестве эталонного спектра при помощи метода наименьших квадратов.

Расчет содержания исследуемого компонента осуществляют в соответствии с выражением:

$$
C_{\text {calc }}=m C_{\text {pegm }},
$$

где коэффициент $m$ определяется путем решения системы линейных уравнений

$$
\left\{D_{\text {meas }}\left(v_{i}\right)=m D_{\text {calc }}\left(v_{i}\right)\right\}
$$

методом наименьших квадратов; $D_{\text {meas }}\left(v_{i}\right)$ - измеренная оптическая плотность спектра исследуемого компонента; $D_{\text {calc }}\left(v_{i}\right)$ - оптическая плотность расчетного спектра; $v_{i}$ - волновое число.

Если относительное стандартное отклонение результата вычисления $C_{\text {calc }}$ от заданного значения $C_{\text {pegm }}$ превышает $0.5 \%$, указанное значение принимается равным результату вычисления $C_{\text {calc }}=C_{\text {pegm }}$ и производится перерасчет. Это сделано, чтобы избежать нелинейности. Наиболее подходящая аппаратная функция прибора подгоняется с использованием коэффициента $m$ (выражение (3)).

Аппаратная функция прибора для ИК фурье-спектрометра, используемого для измерения, может быть выражена в виде аналитических функций, таких как функция Гаусса, функция $\operatorname{sinc}(x)$, соответствующая прямоугольной аподизации, или функция $\operatorname{sinc} 2(x)$, соответствующая треугольной аподизации, или она может быть выражена в табличной форме.

Для определенного прибора выбирается наиболее подходящая функция и производится уточнение ее парамет- 
ров - растяжение/сжатие по оси $X$ (изменение полуширины) и асимметрии (растяжении/сжатии положительной части при пропорциональном сжатии/растяжении отрицательной части).

Численный алгоритм уточнения параметров аппаратной функции основан на поиске таких значений параметров, при которых при расчете содержания определяемого компонента в соответствии с описанным выше алгоритмом сумма квадратов, рассчитанная по формуле

$$
S=\sum_{i}\left(D_{\text {meas }}\left(v_{i}\right)-m D_{\text {calc }}\left(v_{i}\right)\right)^{2},
$$

будет минимальной. Поиск минимума производится путем подбора двух параметров методом покоординатного спуска.

В качестве анализируемой смеси при поиске параметров аппаратной функции берется эталонная смесь $\mathrm{CO}$ в азоте. Стабильность параметров аппаратной функции производится путем контроля молярной доли в эталонной смеси, по которой определялась аппаратная функция.

Относительное отклонение $\delta_{\text {contr }}$ молярной доли, полученное при контрольном измерении $\chi_{\text {contr, }}$ должно отличаться от значения, полученного сразу после определения параметров аппаратной функции $\chi_{\text {init, }}$ не более чем на $0.3 \%$ :

$$
\delta_{\text {contr }}=\frac{\chi_{\text {contr }}-\chi_{\text {init }}}{\chi_{\text {init }}} \cdot 100 \% \leq 0.3 \%
$$

Если $\delta_{\text {contr }}$ не превышает $0.5 \%$, то производится повторное определение параметров аппаратной функции ИК фурье-спектрометра. При превышении $\delta_{\text {contr }}$ значения $0.5 \%$ проводится диагностика неисправности ИК фурье-спектрометра. Критерии $0.3 \%$ и $0.5 \%$ выбраны с учетом достигнутой очень высокой стабильности во времени СО в запаянной кювете.

Результатом вычисления по методу наименьших квадратов является $C_{\text {calc }}$, т. е. полученная молярная доля исследуемого компонента $j$ в газовой смеси по описанной выше методике.

Из-за несоответствия вычисленного спектра с использованием параметров линий БД HITRAN или БД GEISA и экспериментального спектра ПЭГС мы получаем значение концентрации $C_{\text {calc }}$, отличное от $C_{\text {pegm, }}$, т.е. отличное от молярной доли исследуемого компонента $j$ в газовой смеси, записанной в паспорте ПЭГС. Таким образом, при введении коррекции могут быть использованы только расчетные спектры. Поправочные коэффициенты для исследуемого компонента $j$ рассчитываются следующим образом:

$$
K_{j}=\frac{C_{\text {pegm }_{j}}^{\text {meas }}}{C_{\text {pegm }_{j}}^{\text {passp }_{j}}}
$$

где $C_{\mathrm{pegm}_{j}}^{\mathrm{meas}}$ и $C_{\mathrm{pegm}_{j}}^{\mathrm{passp}}-$ молярные доли ПЭГС $j$ в газовой смеси $(\%)$, соответственно измеренная при помощи описанного алгоритма и записанная в паспорте ПЭГС.
Таким образом собирается база данных поправочных коэффициентов $K$ для некоторого диапазона молярной доли анализируемого вещества в газовых смесях.

В результате, измеряя спектр исследуемой газовой смеси, концентрацию анализируемого вещества можно получить без использования ПЭГС, но только с использованием спектроскопических БД HITRAN или GEISA.

Например, молярная доля и массовая концентрация компонентов в газовых средах передается (в настоящее время для $\left.\mathrm{HCl}, \mathrm{CO}, \mathrm{NO}, \mathrm{SO}_{2}\right)$ в ГЭТ 154-2016 от ПЭГС к эталонам сравнения не путем сравнения, но с использованием спектроскопии и спектроскопических БД. Та же самая процедура вводится на местах (в российских городах) для переноса единиц концентрации от эталонов сравнения к рабочим эталонам.

Разработанное Программное обеспечение (ПО) „SpectraLab 2.0“, предназначенное для расчета содержания целевых компонентов $(\mathrm{HCl}, \mathrm{CO}$, $\mathrm{NO}$, $\mathrm{SO}_{2}$ ) в двухкомпонентных газовых смесях на основе спектров, полученных с использованием типового ПО ИК фурье-спектрометров, прошло метрологическую аттестацию во ВНИИМ.

В ходе метрологической аттестации, проведенной в соответствии с ГОСТ Р 8.654-2009 [21], методиками [22-24], стандартом системы менеджмента качества ВНИИМ [25], было подтверждено, что ПО „SpectraLab 2.0“ соответствует предъявляемым к нему требованиям:

- требования к документации ПО;

- требования к идентификации ПО;

- требования к корректности алгоритмов и программ;

- требования к защите программного обеспечения;

- требования к поддержке аппаратных возможностей.

Предоставленная на проверку техническая документация ПО „SpectraLab“ удовлетворяет общим требованиям к документации программного обеспечения по ГОСТ Р 8.654

В качестве входных данных для расчета использовались:

- условия измерений: температура $(T)$, атмосферное давление $(P)$, оптическая длина кюветы $(L)$, аппаратная функция;

- спектры поглощения анализируемых ПЭГС, представленных в табл. 1.

Мы использовали конкретные ПЭГС с конкретными метеорологическими параметрами, которые были подтверждены в процессе ключевых сличений национальных метрологических институтов (НМИ) и включены в базу данных МБМВ [26].

На основе спектров поглощения ПЭГС, полученных в соответствии с требованиями методики измерений [27], проверялась правильность расчета в соответствии с [25]. Относительное отклонение значения молярной доли определяемого компонента $d(X)$, рассчитанного по методике измерений [27], для исследуемых ПЭГС № 1 и № 2 не превышает норматива, приведенного в табл. 2 . 
Таблица 1. ПЭГС, используемые для получения эталонных спектров

\begin{tabular}{c|l|c|c|c|c}
\hline № п/п & Тип эталона & $\begin{array}{c}\text { Определяемый и } \\
\text { фоновый компоненты }\end{array}$ & $\begin{array}{c}\text { Молярная доля } \\
\text { компонента } X, \%\end{array}$ & $\begin{array}{c}\text { Доверительная абсолютная } \\
\text { погрешность } \pm \Delta, \% \text { (при } P=0.99)\end{array}$ & $\begin{array}{c}\text { Относительная } \\
\text { погрешность } \pm \delta, \%\end{array}$ \\
\hline 1 & Хд 2.706.141-ЭТ8 & $\mathrm{CO}+\mathrm{N}_{2}$ & 0.4430 & 0.0013 & 0.3 \\
2 & Хд 2.706.136-ЭТ20 & $\mathrm{CO}+\mathrm{N}_{2}$ & 4.870 & 0.007 & 0.14
\end{tabular}

Таблица 2. Относительное отклонение значения молярной доли определяемого компонента

\begin{tabular}{c|c|c|c|c}
\hline $\begin{array}{c}\text { № ПЭГС } \\
\text { по таблице 4.1 }\end{array}$ & $\begin{array}{c}\text { Определяемый и } \\
\text { фоновый компоненты }\end{array}$ & $\begin{array}{c}\text { Молярная доля } \\
\text { компонента } X, \%\end{array}$ & $d(X)$ & Норматив $d(X)$ \\
\hline 1 & $\mathrm{CO}+\mathrm{N}_{2}$ & 0.4441 & 0.24 & 0.5 \\
2 & $\mathrm{CO}+\mathrm{N}_{2}$ & 4.876 & 0.12 & 0.5
\end{tabular}

Кроме того, мы исследовали точность шкалы волновых чисел ИК фурье-спектрометра FSM 1201 [28] с использованием пленки из полистирола для получения спектра. Точность шкалы волновых чисел составляет $0.07 \mathrm{~cm}^{-1}$, что соответствует требованиям программы испытаний.

\section{Результаты}

Мы разработали методику измерения молярной доли компонентов $\left(\mathrm{HCl}, \mathrm{CO}, \mathrm{NO}, \mathrm{SO}_{2}\right)$ в двухкомпонентных газовых смесях в баллонах под давлением с помощью ИК фурье-спектроскопии. Эта процедура используется для передачи единиц от ГЭТ 154-2016 к рабочим эталонам.

Смесь газа из баллона под давлением нагнетается в газовую кювету, установленную в ИК фурьеспектрометре, для регистрации спектра пропускания в спектральном диапазоне $400-4000 \mathrm{~cm}^{-1}$. С помощью ПO „SpectraLab 2.0“ полученный спектр пропускания сравнивается с расчетными спектрами пропускания, полученными на основе спектроскопической БД HITRAN или GEISA в заданных условиях измерения (значение молярной доли исследуемого компонента, атмосферное давление, температура, длина оптического пути кюветы и аппаратная функция) и рассчитывается молярная доля исследуемого компонента. Полученное значение корректируется с использованием поправочного коэффициента $K$, принимая во внимание систематическое смещение результатов измерения.

Расчет поправочного коэффициента $K$ для каждого компонента проводили в 2-6 точках диапазона измерения инфракрасного ИК фурье-спектрометра FSM 1201. Очевидно, что поправочные коэффициенты будут иметь другие значения для других условий измерения, для другой молярной доли исследуемого компонента, а также для использования другой спектроскопической БД. Значения поправочных коэффициентов при использовании БД HITRAN приведены в табл. 3. Показано, что в нашем случае требуемая коррекция измеренной молярной доли
Таблица 3. Значения поправочных коэффициентов $K$

\begin{tabular}{c|c|c}
\hline $\begin{array}{c}\text { Компонентный } \\
\text { состав } \\
\text { газовой смеси }\end{array}$ & $\begin{array}{c}\text { Диапазон молярной } \\
\text { доли определяемого } \\
\text { компонента } \\
\text { в газовых смесях, \% }\end{array}$ & $\begin{array}{c}\text { Значение } \\
\text { поправочного } \\
\text { коэффициента } K\end{array}$ \\
\hline $\mathrm{HCl}+\mathrm{N}_{2}$ & $0.10-0.30$ & 1.054 \\
$\mathrm{CO}+\mathrm{N}_{2}$ (воздух) & $0.1-8.0$ & 1.050 \\
$\mathrm{NO}+\mathrm{N}_{2}$ (воздух) & $0.2-5.0$ & 1.056 \\
$\mathrm{SO}_{2}+\mathrm{N}_{2}$ (воздух) & $0.05-7.5$ & 1.036
\end{tabular}

варьируется от $3.6 \%$ для $\mathrm{SO}_{2}+\mathrm{N}_{2}$ (воздух) до $5.6 \%$ для $\mathrm{NO}+\mathrm{N}_{2}$ (воздух).

Относительная суммарная стандартная неопределенность измерения молярной доли определяемого компонента в газовой смеси рассчитывалась по формуле:

$$
u_{s}^{0}=\sqrt{\left(u_{\bar{X}_{\text {meas }}}^{0}\right)^{2}+\left(u_{K}^{0}\right)^{2}},
$$

где $u_{\bar{X}_{\text {meas }}}^{0}$ - относительная стандартная неопределенность среднего значения молярной доли компонента в анализируемой газовой смеси, рассчитываемая как стандартное отклонение 3 параллельных определений по формуле

$$
u_{\bar{X}_{\text {meas }}}^{0}=\frac{S}{\bar{X}_{\text {meas }} \sqrt{n}}=\frac{1}{\bar{X}_{\text {meas } \sqrt{n}}} \sqrt{\frac{\sum_{i=1}^{n}\left(X_{\text {meas }_{i}}-\bar{X}_{\text {meas }}\right)^{2}}{n-1}},
$$

где $n-$ количество параллельных определений по формуле (7), $u_{K}^{0}$ - относительная суммарная стандартная неопределенность поправочного коэффициента $K$, рассчитываемая по формуле

$$
u_{K}^{0}=\sqrt{\frac{\delta_{\mathrm{pegm}}^{2}}{3}+\left(\frac{S_{K}}{m}\right)^{2}},
$$

где $\delta_{\text {pegm }}-$ относительная расширенная неопределенность ПЭГС при $k=3(2) ; S_{K}-$ связанное с $K$ относи- 
Таблица 4. Относительная расширенная неопределенность результатов измерений

\begin{tabular}{|c|c|c|}
\hline $\begin{array}{l}\text { Компонентный состав } \\
\text { газовой смеси* }\end{array}$ & $\begin{array}{l}\text { Диапазон измерений молярной доли } \\
\text { определяемого компонента в ГС, \% }\end{array}$ & $\begin{array}{c}\text { Относительная расширенная неопределенность } \\
\text { измерений }(\text { при } n=3, k=2) U, \%^{* *}\end{array}$ \\
\hline $\mathrm{HCl}+\mathrm{N}_{2}$ & от 0.10 до 0.30 & 2 \\
\hline $\mathrm{CO}+\mathrm{N}_{2}$ (воздух $)$ & $\begin{array}{l}\text { От } 0.10 \text { до } 0.50 \text { включительно } \\
\text { Свыше } 0.50 \text { до } 8.0 \text { включительно }\end{array}$ & $\begin{array}{c}0.1 X+1.17 \\
0.0044 X+1.11\end{array}$ \\
\hline $\mathrm{NO}+\mathrm{N}_{2}$ (воздух $)$ & От 0.20 до 5.0 & $0.0201 X+1.01$ \\
\hline $\mathrm{SO}_{2}+\mathrm{N}_{2}$ (воздух $)$ & $\begin{array}{c}\text { От } 0.05 \text { до } 0.10 \text { включительно } \\
\text { Свыше } 0.10 \text { до } 7.5 \text { включительно }\end{array}$ & $\begin{array}{c}-4.29 X+0.82 \\
0.4\end{array}$ \\
\hline
\end{tabular}

Примечание.

* Определяемый и фоновый компоненты.

** Соответствует доверительным границам относительной суммарной погрешности измерений $\pm \delta, \%$ (для доверительной вероятности $P=0.95$ ). $k$ - коэффициент охвата.

тельное отклонение, рассчитанное по формуле:

$$
S_{K}=\frac{100}{K} \sqrt{\frac{\left(K_{\max }-K\right)^{2}}{3}} .
$$

Относительная расширенная неопределенность:

$$
U^{0}=2 u_{C}^{0}
$$

Относительная расширенная неопределенность результатов измерений представлена в табл. 4.

\section{Выводы}

Таким образом, полученные результаты дают возможность введения универсальных ИК фурье-спектрометров для аттестации широкого спектра поверочных газовых смесей с использованием спектроскопических БД HITRAN или GEISA. Отсюда следует, что использование разработанного метода и ИК фурье-спектрометров не требует ПЭГС для аттестации поверочных газовых смесей.

После опытной эксплуатации таких устройств и дальнейшего тестирования разработанного ПО и методов измерения будут проводиться соответствующие корректировки и обновления для большого количества газовых смесей.

Одним из наиболее важных эффектов при использовании спектроскопических БД и универсальных ИК фурьеспектрометров для аттестации стандартных образцов состава газовых смесей является значительное сокращение количества и номенклатуры дорогих и трудоемких для производства газовых смесей - ПЭГС и эталонов сравнения.

Внедрение спектроскопических баз данных, спектральных измерительных приборов, а также разработка методик выполнения измерений и программного обеспечения позволит проводить удаленную передачу единиц измерений от ГЭТ 154-2016 к коммерчески доступным газовым смесям на основе методов спектроскопии в ИК области спектра. Подытоживая опыт ключевых сличений национальных метрологических институтов (НМИ) под эгидой МБМВ [19] и экспериментального использования параметров спектральных линий в качестве эталона, для некоторых проектов МБМВ могут быть сделаны следующие выводы [2]:

- для достижения лучшей точности спектроскопических БД необходимо изменить нынешний формат сбора данных от различных групп ученых, работающих с использованием различных измерительных приборов. Формат сбора данных должен быть ориентирован на создание бюджета неопределенности спектроскопических параметров газов.

- должно быть организовано взаимодействие спектроскопических БД и калибровочных и измерительных возможностей НМИ, представленных в БД МБМВ [29].

- существует необходимость в дальнейшей разработке спектральных методов расчета для улучшения прослеживаемости измерений.

\section{Приложение. Расчет спектра пропускания с использованием лоренцева контура линии}

Коэффициент пропускания определяемого компонента для волнового числа $v$ с использованием параметров линий, приведенных в базе данных HITRAN, определяется по формуле

$$
\tau_{m}(v)=e^{-k(v) x_{\mathrm{set}} L},
$$

где $v-$ волновое число, $k(v)-$ спектральный коэффициент поглощения определяемого компонента; $L-$ длина оптического пути в измерительной кювете, $\mathrm{cm}$; $x_{\text {set }}$ заданная молярная доля определяемого компонента.

Спектральный коэффициент поглощения $k(v)$ рассчитывается по формуле

$$
k(v)=\sum_{i=1}^{n} \sigma_{i}(v) \frac{P}{k T} 10^{-6},
$$

где $T$ - температура газа в измерительной кювете, К; $P$ - давление газа в измерительной кювете, Ра; 
$\sigma_{i}(v)$ - сечение поглощения $i$-й линии в расчете на молекулу, $\mathrm{cm}^{2}$, которое выражается с учетом лоренцева контура линии формулой

$$
\sigma_{i}(v)=\frac{1}{\pi} \frac{S_{i} \alpha_{i}}{\alpha_{i}^{2}+\left(v-v_{i}\right)^{2}},
$$

где $S_{i}$ - интенсивность линии в расчете на молекулу, $\mathrm{cm}, \alpha_{i}$ - полуширина $i$-й линии, $\mathrm{cm}^{-1}, v_{i}-$ положение центра $i$-й линии, $\mathrm{cm}^{-1}$.

Положение центра линии $v_{i}$ рассчитывается по формуле

$$
v_{i}=v_{0 i}+\delta_{i} \frac{P}{P_{0}}
$$

где $P_{0}=101325 \mathrm{~Pa} ; v_{0 i}-$ положение центра $i$-й линии для вакуума, указанное в базе HITRAN, $\mathrm{cm}^{-1}$; $\delta_{i}$ - коэффициент, описывающий смещение центра $i$ й линии, обусловленное давлением, указанный в базе HITRAN, $\mathrm{cm}^{-1} / \mathrm{atm}$.

Интенсивность и полуширина линии имеют зависимость от температуры и давления и рассчитываются по формулам

$$
S_{i}=S_{i 0}\left(\frac{T_{0}}{T}\right)^{Q} \exp \left[\left(1.439 E_{i} \frac{T-T_{0}}{T T_{0}}\right)\right],
$$

где $S_{i 0}-$ интенсивность $i$-й линии для температуры $T_{0}=296 \mathrm{~K}$, указанная в базе HITRAN, $\mathrm{cm}^{2} \mathrm{~cm}^{-1} ; E_{i}-$ энергия нижнего уровня $i$-й линии, указанная в базе HITRAN, $\mathrm{cm}^{-1} ; Q-$ температурный коэффициент $(Q=1$ - для линейных молекул и $Q=1.5-$ для нелинейных молекул)

$$
\alpha_{i}=\left[(1-X) \alpha_{i}^{\mathrm{air}}+X \alpha_{i}^{\mathrm{s}}\right]\left(\frac{T_{0}}{T}\right)^{w_{i}}\left(\frac{P}{P_{0}}\right),
$$

где $\alpha_{i}^{\text {air }}-$ уширение $i$-й линии воздухом, указанное в базе HITRAN, $\mathrm{cm}^{-1} / \mathrm{atm} ; \alpha_{i}^{\mathrm{s}}-$ самоуширение $i$-й линии, указанное в базе HITRAN, $\mathrm{cm}^{-1} / \mathrm{atm} ; w_{i}-$ температурный коэффициент для $i$-й линии, указанный в базе HITRAN, $\mathrm{cm}^{-1}$.

Диапазон волновых чисел и шаг по шкале волновых чисел, для которых производится расчет, может быть задан пользователем.

Расчетный коэффициент спектра пропускания определяемого компонента производится с помощью свертки монохроматического спектра пропускания с аппаратной функцией прибора, на котором аттестуется смесь, по формуле

$$
\tau_{\text {calc }}(v)=\frac{\int_{v_{1}}^{\nu_{2}} \tau_{m}(v) A\left(v^{\prime}-v\right) d v^{\prime}}{\int_{v_{1}}^{v_{2}} A\left(v^{\prime}-v\right) d v^{\prime}},
$$

где $A(v)$ - аппаратная функция спектрометра; $\left[v_{1}, v_{2}\right]$ диапазон, на котором задана аппаратная функция.

Свертка производится с использованием алгоритма быстрого преобразования Фурье. Аппаратная функция может быть задана как аналитически, так и в табулированном виде.

\section{Список литературы}

[1] Konopelko L.A., Beloborodov V.V., Rumiantsev D.V., Selukov D.V. // International Congress of Metrology, 2013. 7-10 October. Paris. 4 pages.

http://cfmetrologie.edpsciences.org/articles/metrology/pdf/ 2013/01/metrology_metr2013_10009.pdf

[2] Konopelko L.A., Beloborodov V.V., Rumiantsev D.V. // 11th ASA Conference (united with the 12th HITRAN Conference) University of Reims. 2012. August 29-31. http:/www.univ-reims.fr/site/evenement/asa-hitran/ gallery_files/site/1/1697/1713/28177/36574.pdf

[3] Konopelko L.A., Beloborodov V.V., Rumiantsev D.V., Selukov D.V. // GEISA Workshop. 2014. 3-4 June, Paris, P. 45. http://www.lmd.jussieu.fr/geisa2014/uploads/images/ NJ_all_abstracts1006.pdf

[4] Features and problems of metrological traceability of gas mixtures using UV absorption, FTIR, and CRD spectroscopy. International Congress of Metrology, 2017. 19-21 September. Paris. 3 pages.

https:/cfmetrologie.edpsciences.org/articles/metrology/pdf/ 2017/01/metrology_metr2017_16002.pdf

[5] Pickett H.M., Poynter R.L., Cohen E.A., Delitsky M.L., Pearson J.C., Muller H.S.P. // JQSRT. 1998. V. 60. P. 883.

[6] Müller H.S.P., Schlöder F., Stutzki J., Winnewisser G. // J. Mol. Struct. 2005. V. 742. P. 215.

[7] Rothman L.S. et al. // JQSRT. 2013. V. 130. P. 4.

[8] Jacquinet-Husson N. et al. // JQSRT. 2011. V. 112. P. 2395.

[9] MPI-Mainz-UV-VIS Spectral Atlas [Электронный pecypc]. Режим доступа: http://joseba.mpch-mainz.mpg.de/ spectral_atlas

[10] NIST Standard Reference Database 79 [Электронный ресурс]. Режим доступа: http://www.nist.gov/srd/nist79.cfm

[11] Beloborodov V.V. // Applied Optics. 2002. V. 41. N 18. P. 3517.

[12] Bailey J. // School of Physics, University of New South Wales, NSW 2052, Australia. http://arxiv.org/ftp/arxiv/papers/ 0901/0901.3869.pdf

[13] Kolokutin G.E., Fomin B.A. // Current problems in remote sensing of the Earth from space (CPRSES). 2014. V. 11. N 3. P. 278. http://jr.rse.cosmos.ru/article.aspx?id=1318\&lang=eng

[14] Guide EBRAHIM/CITAC „Quantitative Uncertainty in Analytical Measurement“. [Электронный pecypc]. Режим доступа: http://www.citac.cc/QUAM2000-1.pdf

[15] Guide to the expression of uncertainty in measurement [Электронный ресурс]. Режим доступа: http://www.bipm.org/ utils/common/documents/jcgm/JCGM_100_2008_E.pdf

[16] Stephanie Bell. A Beginner's Guide to Uncertainty of Measurement [Электронный ресурс]. Режим доступа: https://www.wmo.int/pages/prog/gcos/documents/ gruanmanuals/UK_NPL/mgpg11.pdf

[17] ГОСТ 8.578-2008 Государственная система обеспечения единства измерений (ГСИ). Государственная поверочная схема для средств измерений содержания компонентов в газовых средах.

[18] Другов Ю.С., Конопелько Л.А., Попов О.Г. Контроль загрязнения воздуха жилых помещений, офисов, административных и общественных зданий. Санкт-Петербург: Наука, 2013. С. 302. Глава 5.3.2. Эталонная база РФ в области анализа газов.

[19] Flores E. et al. // Metrologia. 2013. V. 50. Tech. Suppl. 08011, $97 \mathrm{p}$. 
[20] Gasmet Technologies Group. IR Spectrum Collection. [Электронный ресурc]. Режим доступа: http:/www.gasmet.com/ technology/spectra

[21] ГОСТ Р 8.654-2009 Требования к программному обеспечению средств измерений. Основные положения.

[22] МИ 2955-2010 Типовая методика аттестации программного обеспечения средств измерений.

[23] МИ 2174-91 Аттестация алгоритмов и программ обработки данных при измерениях.

[24] МИ 3286-2010 Рекомендация. Проверка защиты программного обеспечения и определение ее уровня при испытаниях средств измерений в целях утверждения типа.

[25] СК 02-30-2007 Программное обеспечение обработки данных при измерениях. Общие положения и порядок проведения метрологической аттестации. Стандарт Системы менеджмента качества ВНИИМ, СПб, 2007. (Слаев B.A., Звягин Н.Д., Чуновкина А.Г.).

[26] Calibration and Measurement Capabilities. Gases, Russian Federation, VNIIM (D.I. Mendeleyev Institute for Metrology, Rosstandart) [Электронный pecypc]. Режим доступа: http://kcdb.bipm.org/AppendixC/QM/RU/QM_RU_4.pdf

[27] СК03/242-4.1.1.2.9-2012 Методика измерения молярной доли компонентов в газовых смесях.

[28] ИК фурье-спектрометры ФСМ. Инфраспек, СанктПетербург, Россия. [Электронный ресурс]. Режим доступа: http://www.infraspek.ru/

[29] BIPM. KCDB. Calibration and Measurement Capabilities. Chemistry. [Электронный ресурс]. Режим доступа: $\mathrm{http} / / \mathrm{kcdb}$.bipm.org/appendixC/search.asp?met=QM\&reset=1 